\title{
ANTOCIANINAS EN CORONTAS Y EXTRACTOS DE MAÍZ MORADO (Zea Mays L) "INIA 615" CONSERVADOS EN ANAQUEL
}

\author{
Samuel Cerro-Ruiz ${ }^{* a}$, Leonardo Espillico-Cormilluni ${ }^{\mathrm{b}}$
}

\begin{abstract}
RESUMEN
Se identificaron y cuantificaron por cromatografía UPLC antocianinas en corontas de maíz morado (Zea mays L) "INIA 615" o "Negro Canaán" y sus extractos conservados en envases de vidrio en anaquel. Se aplicó el método del $\mathrm{pH}$ diferenciado para antocianinas totales y el Diseño Factorial de Variables Mixtas $3 \times 2^{\wedge} 2$ con 14 tratamientos, utilizando el software Statgraphics Centurión XVII para el análisis estadístico, siendo las variables independientes: a) tiempo de almacenaje/muestro: 15, 52 y 90 días; b) envases ámbar, verde e incoloro con iluminación permanente de $470 \mathrm{lux} / \mathrm{m}^{2}$; c) $\mathrm{pH} \mathrm{3,3,5} \mathrm{y} \mathrm{4,} \mathrm{mientras} \mathrm{que} \mathrm{la} \mathrm{variable}$ dependiente fue la degradación de antocianinas totales. En corontas se encontró $12,98 \mathrm{mg} / \mathrm{g}$ de antocianinas totales siendo 4,94 mg/g de cianidina-3-glucósido y 3,38 mg/g de cianidina-3(6"malonilglucósido). En los extractos se encontraron principalmente cianidina-3-glucósido (93,56 $\mu \mathrm{g} / \mathrm{mL})$ y cianidina-3-(6"malonilglucósido) $(48,15 \mu \mathrm{g} / \mathrm{mL})$. Las variables tuvieron un nivel de significancia $\mathrm{p}<0,05$. A 90 días ambas cianidinas se degradaron: cianidina-3glucósido (69,63\%, ámbar, pH 3; 79,05\%, incoloro, $\mathrm{pH} 4)$ y cianidina-3-(6”malonilglucósido) (91,61\%, ámbar, $\mathrm{pH} \mathrm{3;} \mathrm{95,35 \%} \mathrm{incoloro,} \mathrm{pH} 4)$.
\end{abstract}

Palabras clave: Antocianinas, cianidina-3-glucósido, corontas, "INIA 615”, maíz morado.

\section{ANTHOCYANINS IN THE COBS AND EXTRACTS OF PURPLE CORN (Zea Mays L) "INIA 615" PRESERVED UNDER SHELF}

\begin{abstract}
Anthocyanins were identified and quantified by UPLC in purple corn cobs (Zea mays L), also called "INIA 615" or "Negro Canaán", and their extracts were preserved in glass containers on a shelf. The differentiated $\mathrm{pH}$ method and $3 \times 2^{\wedge} 2$ Mixed Factorial Design with 14 fact treatments were applied to total anthocyanins using the Statgraphics Centurion XVII for statistical analysis and taking into account the degradation of total anthocyanins as the

\footnotetext{
*a Departamento Académico de Ingeniería en Industrias Alimentarias-Facultad de Ciencias Agropecuarias. Universidad Nacional Jorge Basadre Grohmann, Av. Miraflores s/n, Tacna, Tacna, Tacna, Perú. scerror@unjbg. edu.pe. ID ORCID: https://orcid.org/0000-0001-7359-3503.

b Laboratorio de Operaciones Unitarias Escuela de Ingeniería en Industrias Alimentarias - Facultad de Ciencias Agropecuarias - Universidad Nacional Jorge Basadre Grohmann. Av. Miraflores s/n, Tacna, Tacna, Tacna, Perú. ID ORCID: https://orcid.org/0000-0003-1717-8318.
} 
dependent variable as well as the following independent variables: a) storage/sampling time: 15, 52, and 90 days; b) amber, green, and colorless containers with permanent lighting of $470 \mathrm{lux} / \mathrm{m}^{2}$; and c) $\mathrm{pH} \mathrm{3,} \mathrm{3.5,} \mathrm{and} \mathrm{4.} \mathrm{In} \mathrm{crowns,} 12.98 \mathrm{mg} / \mathrm{g}$ of total anthocyanins were found, including $4.94 \mathrm{mg} / \mathrm{g}$ of cyanidin-3-glucoside, and $3.38 \mathrm{mg} / \mathrm{g}$ of cyanidin-3-(6"malonylglucoside). Extracts mostly contained $93.56 \mu \mathrm{g} / \mathrm{mL}$ of cyanidin-3-glucoside, and $48.15 \mu \mathrm{g} / \mathrm{mL}$ of cyanidin-3-(6"-malonylglucoside). Variables had a significance level of $\mathrm{p}<$ 0.05. At 90 days, both cyanidins were degraded: cyanidin-3-glucoside $(69.63 \%$, amber, $\mathrm{pH}$ 3; 79.05\%, colorless, $\mathrm{pH} 4$ ), and of cyanidin-3-(6"-malonylglucoside) (91.61\%, amber, pH 3; 95.35\%, colorless, $\mathrm{pH} 4$ ).

Keywords: anthocyanins, cyanidin-3-glucoside, corn cobs, "INIA 615", purple corn.

\section{INTRODUCCIÓN}

En el Perú es común la producción y consumo de chichas, refrescos y postres elaborados a partir de maíz morado (Zea mays L), teniendo esta tradición desde tiempos pre incas, por lo que se conocen distintas variedades de maíz morado que difieren entre sus características como el color de la planta, la dimensión de la coronta, el número de hileras de granos, hasta el color del grano y de la mazorca, siendo esta disparidad consecuente a la presencia de genes mayores y menores en los cromosomas que posee cada variedad ${ }^{1}$, observándose en las corontas de la variedad "INIA 615" conocido como Negro Canaán que tienen forma cilíndrica, delgada, color morado oscuro.

Actualmente, existe el incremento del interés científico por la obtención de antocianinas y sus procedimientos de extracción a partir de recursos vegetales, debido a que su inclusión en la dieta diaria tendría un alto potencial de reducción de la oxidación celular ${ }^{2}$, ya que la antocianina mayoritaria presente en el maíz morado es la cianidin-3-glucósido siendo esta la que previene el daño hepático producido por la ingesta de bebidas alcohólicas o por una inapropiada alimentación. Esto tiene relación con los beneficios a la salud que trae el consumo de cereales o alimentos funcionales adicionados con pigmentos coloreados, provenientes de antocianinas, manifestándose así en efectos anticancerígenos, regulatorios de la glucosa, de neuroprotección y retardo del envejecimiento en el organismo humano ${ }^{3}$.

Cabe resaltar la existencia de estudios que han encontrado una significativa influencia del $\mathrm{pH}$, la luz, temperatura y estructura en condiciones ácidas sobre la perdurabilidad del color en las antocianinas ${ }^{4}$. Además, el efecto de la luz ${ }^{5}$ incidiría en la estabilidad de las antocianinas y otros componentes del vino tinto, siendo el tipo de color del envase de vidrio (transparente, verde y marrón) el factor determinante. No obstante, existe una escasa investigación sobre la conservación y estabilidad de bebidas en forma de extractos de maíz morado conservados en envases de vidrio en condiciones de anaquel.

De tal manera que en la presente investigación se buscó, empleando el método del pH diferenciado, identificar y cuantificar por la técnica de Cromatografía Líquida de Ultra Rendimiento (UPLC), las principales antocianinas y sus derivados presentes en extractos de 
maíz morado "INIA 615", procesados a valores de $\mathrm{pH}$ 3, 3,5 y 4 conservados ambientalmente en envases de vidrio colores ámbar, verde e incoloro hasta por 90 días con luz de fluorescentes que emitían 470 luxes $/ \mathrm{m}^{2}$.

\section{PARTE EXPERIMENTAL}

\section{Material}

Las corontas secas de maíz morado (Zea mays L.) INIA 615, fueron obtenidas del Instituto de Innovación Agraria (INIA) siendo producidas en su Estación Experimental de Uraca - Corire (429 msnm) parte baja del valle del río Majes, Provincia de Castilla, Región Arequipa, sur del Perú.

\section{Molienda, tamizado de coronta y preparación de solventes}

Se utilizaron 5,0 kg de corontas de maíz morado "INIA 615" que fueron masados en balanza Ohaus Corporation, USA; luego fueron molidas en un molino de martillos marca PeruMinox modelo MM4. Se masaron 2,5 Kg que fueron pasados por los tamices $\mathrm{N}^{\circ} 18(1,00 \mathrm{~mm}), 20$ $(0,85 \mathrm{~mm})$ y $140(0,106 \mathrm{~mm})$ marca W S Tyler, USA Standard Testing Sieve, descartándose las partículas del último tamiz. Las corontas de maíz presentaron una humedad de $8 \%$. La muestra fue pulverizada y homogenizada con solución microbicida combinada de benzoato de sodio y sorbato de potasio grado alimentario cada uno al 0,05\% diluidas en $0,5 \mathrm{~L}$ de etanol $70 \%$ vol. rebajado a $20 \%$ vol, de Laboratorio Alkofarma EIRL, Perú y envasada en bolsas de polietileno Z-ploc de $100 \mathrm{~g}$, puestas en congelación a $-18^{\circ} \mathrm{C}$ por ocho días para mantenerla preservada de microorganismos e insectos hasta el inicio de la fase experimental.

2.1. Acidificación del agua para extracción: Las soluciones acuosas ácidas de $\mathrm{pH} 3,3,5$ y 4 fueron logradas con diluciones en agua destilada de 0,3256, 0,0610 y 0,0120 g/L de ácido cítrico grado alimentario y controlado con pH-metro marca Ohaus tipo ST 20.

2.2. Dilución de coronta - agua y homogenizado: La relación coronta molida - agua fue de $1: 25$ partes $^{6}$.

\section{Extracción de pigmentos de antocianina en baño maría}

El volumen del extracto a pH 3 fue repartido en seis beakers de $600 \mathrm{~mL}$ de capacidad. Igual distribución se realizó con el extracto a pH 4. En el caso del pH 3.5, se usaron dos beakers de $600 \mathrm{~mL}$. Los 14 vasos de extracción se colocaron en dos equipos de baño maría marca GFL, tipo 1032 para un proceso en caliente con valores de temperatura controlada7 de $75^{\circ} \mathrm{C}$ y durante 150 minutos1. Se realizó agitación manual constante.

\section{Filtrado y conservación}

Los extractos fueron filtrados usando papel Watman $\mathrm{N}^{\circ} 1$ y embudo Büchner, con ayuda de una bomba de vacío. Para la estabilidad microbiológica de los extractos se adicionó benzoato de sodio y sorbato de potasio cada uno en solución al $0,05 \%$. 


\section{Envasado y sellado}

El envasado manual en caliente se realizó en envases de vidrio incoloro de capacidad de 300 $\mathrm{mL}$ con tapas twist off, envases de color verde de capacidad de $250 \mathrm{~mL}$ con tapas pilfer y envases de color ámbar de capacidad de $330 \mathrm{~mL}$ con tapas corona. Al término se procedió al sellado.

\section{Almacenaje en anaquel}

El almacenaje de los extractos en anaquel se realizó en ambiente cerrado, amplio, ventilado, anexo al Laboratorio de Operaciones Unitarias, Escuela Profesional de Ingeniería en Industrias Alimentarias, Universidad Nacional Jorge Basadre Grohmann en Tacna, durante los meses de abril a junio, en condiciones ambientales de temperatura $\left(9,1{ }^{\circ} \mathrm{C}-27,9{ }^{\circ} \mathrm{C}\right)$ y humedad $(68,2 \%-97,4 \%)^{8}$. La iluminación fue con cuatro pantallas y ocho tubos fluorescentes de luz blanca (320 watts) suspendidas a 1,10 m sobre las muestras que generaban una iluminación de 470 luxes $/ \mathrm{m}^{2}$ durante 24 horas hasta por 90 días. Las muestras fueron retiradas en tres lotes a los 15,52 y 90 días de almacenaje.

\section{Preparación y envío de muestras al laboratorio}

Cumplidos los tiempos de almacenaje en anaquel, según el diseño factorial experimental, se retiraron $15 \mathrm{~mL}$ de las muestras seleccionadas y se envasaron asépticamente en tubos plásticos con tapa siendo puestos en congelación a $-18^{\circ} \mathrm{C}$ por 48 horas para su conservación. Luego se empacaron con bolsitas congelantes en una caja isotérmica (cooler) y enviadas vía aérea al Instituto de Biotecnología (IBT) de la Universidad Nacional Agraria La Molina (UNALM) en Lima, Perú.

\section{Identificación y cuantificación de antocianinas empleando UPLC}

Para los análisis cromatográficos de identificación y cuantificación el Instituto de Biotecnología (IBT) de la UNALM empleó un sistema de clase H de Cromatografía Líquida de Ultra Rendimiento (UPLC) aplicando un método de análisis adaptado 9 .

Las antocianinas fueron separadas utilizando una columna analítica Acquity UPLC BEH C18 ( $100 \times 2,1 \mathrm{~mm}$ i.d., tamaño de partícula de $1,7 \mu \mathrm{m}$, Waters) y protegido con una pre columna Absty UPLC BEH C18 Van Guard ( 5 x 2,1 mm i.d., tamaño de partícula de 1,7 mm, Waters). El volumen de inyección en las corridas fue de 2 ul. Los solventes fueron: Milli Q (5\% ácido fórmico) [A] y Acetonitrilo 100 \% [B]. La Marca del equipo es Acquity de Waters, Milford, Mass., U.S.A., equipado con un detector de matriz de diodos (PDA), una bomba cuaternaria, un muestreador automático y controlado por el software Empower III (Waters). El flujo y tiempo de corrida: $0,15 \mathrm{~mL} / \mathrm{min}$. Temperatura de columna: $30^{\circ} \mathrm{C}$ y de la muestra: $5^{\circ} \mathrm{C}$. La columna fue Acquity UPLC BEH C18 1.7 um $(2,1 \times 100 \mathrm{~mm})$ con matriz de fotodiodos (PDA).

En cuanto a la cuantificación de antocianinas el Laboratorio (IBT) de la UNALM disponía de los patrones necesarios con los cuales preparó soluciones a diferentes concentraciones para construir curvas de calibración que sirvieron de referencia para contrastar con los resultados de las corridas cromatográficas de las muestras analizadas. 


\section{Diseño experimental y análisis estadístico}

Fue empleado el Diseño Factorial Fraccionado de Variables Mixtas $3 \times 2^{\wedge} 2$ con dos puntos centrales haciendo un total de 14 tratamientos. Las variables independientes fueron: tiempo de almacenaje y muestreo (15, 52 y 90 días); envases de vidrio colores ámbar, verde e incoloro; $\mathrm{pH}$ 3, 3,5 y 4. Variable dependiente las antocianinas identificadas, cuantificadas y degradadas en almacenaje. Las variables relacionadas con dos antocianinas fueron evaluadas por Análisis de Varianza con un grado de significancia $p<0,05$. El análisis estadístico fue desarrollado utilizando el software Statgraphics Centurión XVII y para las figuras se utilizaron las herramientas gráficas del software Microsoft Excel.

\section{RESULTADOS Y DISCUSIÓN}

\section{Antocianinas en coronta de maíz morado}

Los análisis de cromatografía UPLC en coronta de maíz morado "INIA615" revelaron la presencia de ocho antocianinas como se observa en la Tabla 1. La cianidina-3-glucósido fue el componente principal y peonidina-3-(6"etilmalonilglucósido) fue el minoritario. Según la literatura consultada, ${ }^{1,10}$ la presencia de antocianinas en diferentes vegetales puede variar en función de la materia prima, su variedad, la relación soluto-solvente, los solventes utilizados, procesos y parámetros de extracción, tiempo y condiciones de almacenaje.

Tabla 1. Identificación y contenido de antocianinas en coronta de "INIA 615" determinado por UPLC.

\begin{tabular}{clc}
\hline $\mathrm{N}^{\circ}$ & \multicolumn{1}{c}{ Antocianinas } & $\begin{array}{c}\text { Contenido } \\
(\mathrm{mg} / \mathrm{g})\end{array}$ \\
\hline 1 & Cianidina-3-glucósido & 4,94 \\
2 & Pelargonidina-3-glucósido & 1,07 \\
3 & Peonidina-3-glucósido & 1,38 \\
4 & Cianidina-3-(6"malonilglucósido) & 3,38 \\
5 & Pelargonidina-3-(6"malonilglucósido) & 1,22 \\
6 & Peonidina-3-(6"malonilglucósido) & 0,87 \\
7 & Pelargonidina-3-(6"etilmalonilglucósido) & 0,07 \\
8 & Peonidina-3-(6"etilmalonilglucósido) & 0,05 \\
\hline Antocianinas totales & 12,98 \\
\hline
\end{tabular}

Otros investigadores ${ }^{14}$, indican que las antocianinas son abundantes en vegetales, pero los valores más altos se presentan en maíces y camotes morados llegando hasta los $1400 \mathrm{mg} / 100$ $\mathrm{g}$, siendo este valor similar al obtenido en esta investigación para la coronta de maíz morado. Por otra parte, se reportaron6 6altas concentraciones de antocianinas monoméricas (290- 
$1325 \mathrm{mg} / 100 \mathrm{~g}$ MS) en mazorcas de 10 muestras de maíces morados peruanos siendo las principales detectadas la cianidina-3-glucósido, pelargonidina-3-glucósido, peonidina-3glucósido, cianidina-3-(6"malonilglucósido) y otros derivados más. Estos resultados también concuerdan con los resultados obtenidos.

\section{Antocianinas en extractos}

En la Tabla 2 observamos las antocianinas y sus derivados detectados por cromatografía UPLC en los extractos de coronta de maíz morado "INIA 615" durante el almacenaje y según el diseño experimental empleado.

Tabla 2. Identificación y contenido de antocianinas y derivados por UPLC en extractos de coronta de maíz morado (Zea mays L) "INIA 615".

\begin{tabular}{|c|c|c|c|c|c|c|c|c|}
\hline Ensayo & Tiempo & Envase & $\mathrm{pH}$ & $\begin{array}{c}\text { Cianidina-3 } \\
\text { glucósido }\end{array}$ & $\begin{array}{l}\text { Pelargonidi } \\
\text { na-3 } \\
\text { glucósido }\end{array}$ & $\begin{array}{l}\text { Peonidina } \\
-3- \\
\text { glucósido }\end{array}$ & $\begin{array}{l}\text { Cianidina3 } \\
\text { (6"malonil } \\
\text { glucósido) }\end{array}$ & $\begin{array}{l}\text { Pelargonidi } \\
\text { na-3 } \\
\text { (6"malonil } \\
\text { glucósido) }\end{array}$ \\
\hline $\mathrm{N}^{\circ}$ & Días & Color* & Unidad & $\mu \mathrm{g} / \mathrm{mL}$ & $\mu \mathrm{g} / \mathrm{mL}$ & $\mu \mathrm{g} / \mathrm{mL}$ & $\mu \mathrm{g} / \mathrm{mL}$ & $\mu \mathrm{g} / \mathrm{mL}$ \\
\hline 1 & 52 & 2 & 3,5 & 69,39 & 13,25 & 17,2 & 18,66 & 1,09 \\
\hline 2 & 52 & 2 & 3,5 & 58,96 & 9,74 & 13,1 & 15,83 & 0,98 \\
\hline 3 & 90 & 3 & 4 & 41,41 & 10,26 & 3,5 & 6,28 & 1,56 \\
\hline 4 & 15 & 1 & 3 & 93,56 & 22,63 & 18,1 & 48,15 & 13,30 \\
\hline 5 & 15 & 3 & 4 & 75,89 & 16,49 & 12,1 & 36,31 & 8,41 \\
\hline 6 & 90 & 1 & 4 & 59,02 & 15,85 & 6,5 & 10,42 & 2,44 \\
\hline 7 & 15 & 3 & 3 & 84,53 & 17,09 & 15,8 & 43,60 & 11,67 \\
\hline 8 & 52 & 1 & 3 & 79,47 & 15,34 & 21,5 & 23,30 & 2,27 \\
\hline 9 & 15 & 1 & 4 & 85,21 & 20,58 & 15,2 & 39,78 & 9,59 \\
\hline 10 & 52 & 1 & 4 & 68,38 & 11,18 & 17,1 & 18,72 & 1,53 \\
\hline 11 & 90 & 1 & 3 & 60,00 & 16,25 & 7,5 & 11,34 & 2,56 \\
\hline 12 & 52 & 3 & 3 & 57,04 & 8,83 & 13,2 & 17,27 & 1,03 \\
\hline 13 & 52 & 3 & 4 & 52,36 & 7,77 & 11,4 & 14,80 & 9,10 \\
\hline 14 & 90 & 3 & 3 & 45,72 & 11,32 & 4,8 & 8,38 & 2,46 \\
\hline
\end{tabular}

* Colores de envases: 1 ámbar; 2 verde; 3 incoloro.

Respecto de las bajas concentraciones de antocianinas obtenidas en los extractos acuosos acidulados de la experimentación, coinciden con otros investigadores ${ }^{11}$. Ellos hicieron extracciones de antocianinas y compuestos fenólicos en mazorcas de maíz morado ceroso de Tailandia asistida por ultrasonido utilizando como solventes agua pura, etanol-agua (50$50 \%$ ) y etanol (100\%). En el extracto con agua pura obtuvieron tan solo 37,095 ug/g muestra seca. 
Además, en la Tabla 2 se observa que la C-3-G y su derivado C-3-(6"mg) fueron los dos principales constituyentes en los extractos de coronta de maíz morado "INIA 615" conservados en envases de vidrio ámbar e incoloro ambos a $\mathrm{pH} 3$ y 4 . Los extractos en envases verdes, según modelo experimental, sólo fueron dos muestras a 45 días.

Habiéndose encontrado que la cianidina-3-glucósido y la cianidina-3-(6"malonilglucosido) fueron las de mayor presencia, tanto en la materia prima como en los extractos, se decidió enfocar el estudio en estas dos determinando así su degradación nominal y porcentual durante el almacenaje en anaquel ambiental. Los resultados se observan en las Figuras 1 y 2.

\section{Análisis de varianza de antocianinas en extractos}

En las Tablas 3 y 4 se muestran los análisis de varianza para las dos principales antocianinas detectadas en los extractos siendo el tiempo de almacenaje, color del envase de vidrio y valor de $\mathrm{pH}$ las variables más influyentes $(\mathrm{p}<0,05)$.

Tabla 3. Análisis de varianza de cianidina-3-glucósido.

\begin{tabular}{lccccc}
\hline Factor & Suma de Cuadrados & Df & Cuadrado medio & F- Relación & P-Valor \\
\hline A: Tiempo & 0,00221246 & 1 & 0,00221246 & 132,72 & 0,0000 \\
B: Color de envase & 0,00065549 & 1 & 0,00065549 & 39,32 & 0,0008 \\
C: $p H$ & 0,00012065 & 1 & 0,00012065 & 7,24 & 0,0360 \\
AA & $5,2171 \mathrm{E}-05$ & 1 & $5,21709 \mathrm{E}-05$ & 3,13 & 0,1273 \\
AB & $2,2916 \mathrm{E}-05$ & 1 & $2,29164 \mathrm{E}-05$ & 1,37 & 0,2854 \\
AC & $1,7111 \mathrm{E}-05$ & 1 & $1,71113 \mathrm{E}-05$ & 1,03 & 0,3501 \\
BC & $6,49 \mathrm{E}-07$ & 1 & $6,49 \mathrm{E}-07$ & 0,04 & 0,8501 \\
\hline Total error & 0,00010002 & 6 & $1,66703 \mathrm{E} 05$ & & \\
Total(corr.) & 0,00318147 & 13 & & & \\
\hline
\end{tabular}

Tabla 4. Análisis de varianza de cianidina-3-(6"malonilglucosido).

\begin{tabular}{llllll}
\hline Factor & Suma de Cuadrados & Df & Cuadrado medio & F-Relación & P-Valor \\
\hline A: Tiempo & 0,0021589 & 1 & 0,0021589 & 1376,05 & 0,0000 \\
B: Color de envase & 0,0000523754 & 1 & 0,0000523754 & 33,38 & 0,0012 \\
C: pH & 0,0000551694 & 1 & 0,0000551694 & 35,16 & 0,0010 \\
AA & 0,000189554 & 1 & 0,000189554 & 120,82 & 0,0000 \\
AB & $1,058 \mathrm{E}-7$ & 1 & $1,058 \mathrm{E}-7$ & 0,07 & 0,8038 \\
AC & 0,0000199712 & 1 & 0,0000199712 & 12,73 & 0,0118 \\
BC & $3,36675 \mathrm{E}-7$ & 1 & $3,36675 \mathrm{E}-7$ & 0,21 & 0,6595 \\
\hline Total error & 0,00000941348 & 6 & 0,00000156891 & & \\
Total (corr.) & 0,00248583 & 13 & & & \\
\hline
\end{tabular}




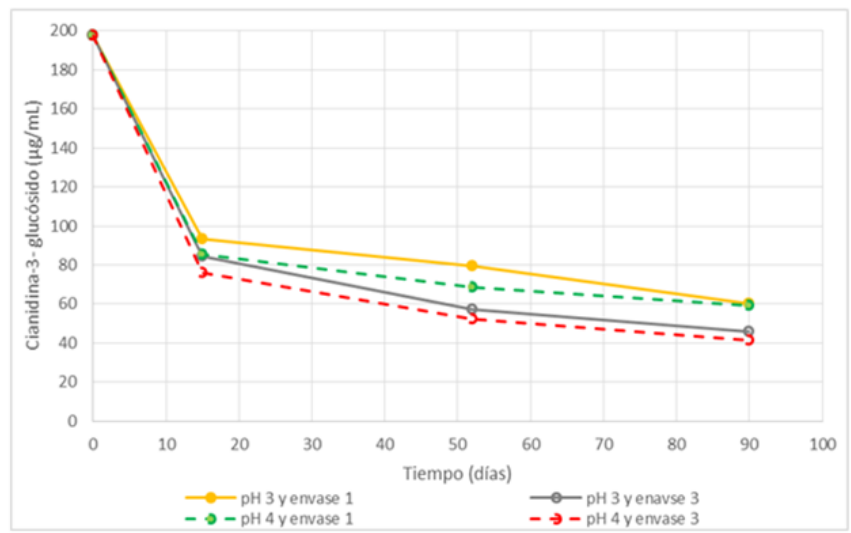

Figura 1. Degradación de cianidina-3-glucósido $(\mu \mathrm{g} / \mathrm{mL})$ en extractos a $\mathrm{pH} 3$ y 4 y envases ámbar (1) e incoloro (3) en condiciones de anaquel.

En el caso de la cianidina 3 glucósido (Figura 1), partiendo de una concentración inicial de $197,6 \mu \mathrm{g} / \mathrm{mL}$ a 90 días de almacenaje en condiciones de anaquel ambiental con luz blanca permanente de fluorescentes $\left(470 \mathrm{lux} / \mathrm{m}^{2}\right)$, se observa que su contenido fue disminuyendo en un rango de $60,0 \mu \mathrm{g} / \mathrm{mL}$ a $41,41 \mu \mathrm{g} / \mathrm{mL}$ generando una pérdida por degradación del $69,63 \%$ para envase ámbar y $\mathrm{pH} 3$, hasta 79,05\% para envase incoloro y $\mathrm{pH} 4$.

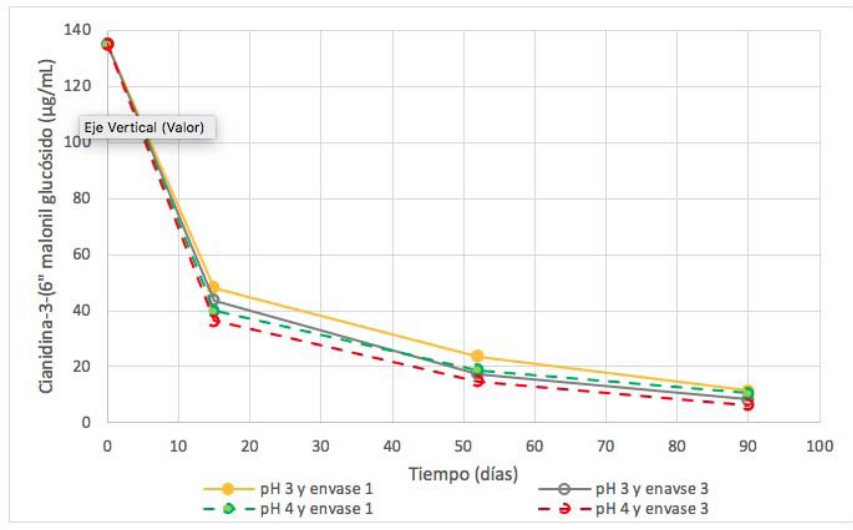

Figura 2. Degradación de cianidina-3-(6"malonilglucósido) $(\mu \mathrm{g} / \mathrm{mL})$ en extractos a pH 3 y 4 y envases ámbar (1) e incoloro (3) en condiciones de anaquel. 
En la Figura 2, para el caso de la cianidina-3-(6"malonilglucósido) presente en los extractos procesados y conservados en los mismos envases, $\mathrm{pH}$ y condiciones, se observa disminución, desde un valor inicial de $135,2 \mu \mathrm{g} / \mathrm{mL}$ hasta $11,34-6,28 \mu \mathrm{g} / \mathrm{mL}$ representando degradaciones entre el $91,61 \%$ para el envase ámbar y pH 3, hasta 95,35\% en envase incoloro y pH 4 . En relación con el análisis de varianza para ambos componentes (Tablas 3 y 4), bajo condiciones de conservación en anaquel descritos, las variables tiempo, protección del color de envase ante la luz y $\mathrm{pH}$, tuvieron un notable nivel de significancia $(\mathrm{p}<0,05)$ siendo, en ese orden, las que influyeron en la inestabilidad de las antocianinas.

Existen investigaciones sobre la degradación ${ }^{12}$ de compuestos fenólicos y capacidad antioxidante in vitro de vinos tintos Syrah y Moscatel almacenados durante un periodo máximo de doce meses en botellas de vidrio colores verde, ámbar y transparente. Los principales resultados fueron la modificación de la tonalidad, disminución de catequinas y más acentuada la antocianina malvidina-3-glucósido, provocados por el tiempo de almacenaje y no por el

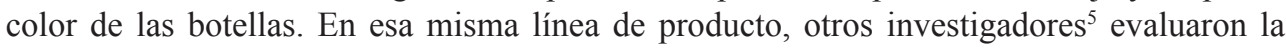
estabilidad de vino tinto almacenado seis meses en botellas transparentes, marrones y verdes con y sin luz artificial reportando diferencias significativas de carácter químico en pigmentos rojos, antocianinas, fenoles totales y mayor envejecimiento para los vinos en botellas transparentes y efectos no significativos para los vinos en botellas marrones y verdes.

Siguiendo con el tema de la disminución o pérdida de antocianinas y compuestos fenólicos ya sea debido a factores de procesamiento y/o de almacenaje, pero esta vez en néctares de agraz (Vaccinium meridionale Swartz), una baya morada con altos contenidos polifenólicos. Se reportó ${ }^{13}$ que a $\mathrm{pH} 3$ se produce una reducción importante $(\mathrm{p}<0,005)$ en el contenido de antocianinas originado primeramente por la pasteurización, coincidiendo en esto con otros investigadores ${ }^{14}$, de que dicho proceso generó una pérdida de $28,5 \%$ en la concentración de antocianinas en jugos de arándanos. Además, se detectó desde el primer día de almacenaje y hasta el día 42, una pérdida constante de antocianinas de 64,28 \%-70,80\%. Estos valores encontrados fueron similares a los de la presente investigación y concuerdan también con lo reportado acerca de que el factor tiempo ${ }^{15}$ y condiciones de almacenaje, afectan la estabilidad de antocianinas. Adicionalmente y con respecto a los efectos del factor luz ${ }^{5,16}$ (ultravioleta) sobre las antocianinas, se han reportado casos en que aun empleando co-pigmentos protectores en la cianidina-3-glucosido, esta llegaba a degradarse en el tiempo.

\section{CONCLUSIONES}

Se confirmó la presencia y se cuantificó la cianidina-3-glucósido y su forma glucosilada y acilada, la cianidin-3-(6"malonilglucósido) tanto en corontas secas como en sus extractos acuosos así como presencia de peonidina-3-glucósido, pelargonidina-3-glucósido y sus derivados. Las variables tiempo de almacenaje, color de envase y $\mathrm{pH}$ tuvieron un nivel de significancia $\mathrm{p}<0,05$. 
Bajo condiciones ambientales de conservación en anaquel hasta un periodo de 90 días, la cianidina-3-glucósido mostró una degradación entre 69,63\% (envase ámbar, pH 3) y 79,05\% (envase incoloro, $\mathrm{pH} 4$ ), mientras que su derivado malónico cianidina-3-(6"malonilglucósido), bajo las mismas condiciones, reportó una degradación del 91,61\% (ámbar, pH 3) y 95,35\% (incoloro, $\mathrm{pH} 4$ ). Los envases color ámbar ofrecieron una relativa protección de las antocianinas frente al tiempo y condiciones de almacenaje con iluminación.

\section{AGRADECIMIENTO}

Los investigadores agradecen al Dr. Efrén Chaparro-Montoya por la revisión del artículo y al Vice Rectorado de Investigación de la Universidad Nacional Jorge Basadre Grohmann de Tacna, por haber seleccionado y financiado esta investigación. Así mismo, a los asistentes administrativo y de laboratorio Bch. Pamela Toledo-Merma y Bch. Néstor Tintaya-Landeo.

\section{REFERENCIAS BIBLIOGRÁFICAS}

1. Alarcón C. Optimización de parámetros de extracción de antocianinas del maíz morado (zea mayz L.) por el método de superficie de respuesta y verificación experimental [Tesis de Maestría]. Ayacucho: Universidad Nacional de San Cristóbal de Huamanga; 2015.

2. Zhou Y, Wang S, Wan T, Huang Y, Pang N, Jiang X, et al. Cyanidin-3-O- $\beta$-glucoside inactivates NLRP3 inflammasome and alleviates alcoholic steatohepatitis via SirT1/NF$\kappa \mathrm{B}$ signaling pathway. Free Radic Biol Med. 2020; 160: 334-341.

3. Zhu F. Anthocyanins in cereals: Composition and health effects. Food Res Int. 2018; 109: 232-49.

4. Khoo HE, Azlan A, Tang ST, Lim SM. Anthocyanidins and anthocyanins: colored pigments as food, pharmaceutical ingredients, and the potential health benefits. Food Nutr Res. 2017; 61: 1361779. doi: 10.1080/16546628.2017.1361779

5. Guerrini L, Pantani O, Politi S, Angeloni G, Masella P, Calamai L, et al. Does bottle color protect red wine from photo-oxidation? Packag Technol Sci. 2019; 32(5): 259265.

6. Jing P, Noriega V, Schwartz S, Giusti M. Effects of Growing Conditions on Purple Corncob (Zea mays L.) Anthocyanins. J Agric Food Chem. 2007; 55(21): 8625-8629.

7. Gorriti-Gutierrez A, Arroyo-Acevedo J, Negrón-Ballarte L, Jurado-Teixeira B, PurizacaLLajaruna H, Santiago-Aquise I, et al. Antocianinas, fenoles totales y actividad antioxidante de las corontas de maíz morado (Zea mays L.): Método de extracción. BLACPMA. 2009; 8(6): 509-518.

8. Servicio Nacional de Meteorología e Hidrología (SENAMHI). Promedio de temperatura normal para Tacna [Internet]. 2019 [Consultado el 23 Sep 2021]. Disponible en: https:// www.senamhi.gob.pe/main.php?dp=tacna\&p=pronostico-detalle

9. De Pascual-Teresa S, Santos-Buelga C, Rivas-Gonzalo J. LC-MS analysis of anthocyanins from purple corn cob. J Sci Food Agric. 2002; 82(9): 1003-1006. 
10. Santos-Buelga C, González-Paramás A. Anthocyanins. En: Encyclopedia of Food Chemistry [Internet]. 2019 [Consultado el 28 Sep 2021]. p. 10-21. Disponible en: https://doi.org/10.1016/B978-0-08-100596-5.21609-0

11. Muangrat R, Pongsirikul I, Blanco P. Ultrasound assisted extraction of anthocyanins and total phenolic compounds from dried cob of purple waxy corn using response surface methodology. J Food Process Preserv. 2018; 42(2): e13447. doi: 10.1111/jfpp.13447

12. De Souza J, de Souza Nascimento A, Linhares M, Dutra M, dos Santos Lima M, Pereira G. Evolution of phenolic compound profiles and antioxidant activity of syrah red and sparkling moscatel wines stored in bottles of different colors. Beverages. 2018; 4(4): 89. doi: 10.3390/beverages4040089

13. Franco YN, Rojano B, Alzate AF, Restrepo CE, Rivero DM, Maldonado ME.Efecto del tiempo de almacenamiento sobre las propiedades fisicoquímicas y antioxidantes de pulpa congelada y liofilizada del fruto de agraz (Vaccinium meridionale Swartz). Vitae. 2016; 23(3): 184-193.

14. Zapata L, Castagnini J, Quinteros C, Carlier E, Jimenez-Veuthey M, Cabrera C. Estabilidad de antocianinas durante el almacenamiento de jugos de arándanos. Vitae. 2016; 23(3): 173-183.

15. Morais H, Ramos C, Forgács E, Cserháti T, Oliviera J. Influence of storage conditions on the stability of monomeric anthocyanins studied by reversed-phase high-performance liquid chromatography. J Chromatogr B. 2002; 770(1-2): 297-301.

16. Bąkowska A, Kucharska AZ, Oszmiański J. The effects of heating, UV irradiation, and storage on stability of the anthocyanin-polyphenol copigment complex. Food Chem. 2003; 81(3): 349-355. 